The Astrophysical Journal, 205:29-43, 1976 April 1

(C) 1976. The American Astronomical Society. All rights reserved. Printed in U.S.A.

\title{
OPTICAL AND INFRARED SPECTROPHOTOMETRY OF 18 MARKARIAN GALAXIES
}

\author{
G. Neugebauer, E. E. Becklin, J. B. OKe, and L. Searle \\ Hale Observatories, California Institute of Technology, Carnegie Institution of Washington \\ Received 1975 August 21; revised 1975 September 17
}

\begin{abstract}
Slit spectra, spectrophotometric scans, and infrared broad-band observations are presented of 18 Markarian galaxies with emission lines. Eight of the program galaxies can be classified as Seyfert galaxies. Arguments are given that thermal, nonthermal, and stellar radiation components are present. Broadly speaking, one group of Seyfert galaxies is characterized both by the presence of a high-density region of gas and by a continuum dominated by nonthermal radiation. The continua of the remaining program Seyferts, which do not have a high density region of gas, are dominated by thermal radiation from dust and a stellar continuum. The 10 galaxies which are not Seyfert galaxies are shown to be examples of extragalactic $\mathrm{H}$ II regions; there is evidence for thermal emission from dust being present at $10 \mu$ in four of these galaxies.
\end{abstract}

Subject headings: galaxies: Seyfert - infrared: sources - nebulae: general

\section{INTRODUCTION}

The interest in peculiar galaxies such as Seyfert and $\mathrm{N}$-type galaxies has led to studies of substantial numbers of peculiar galaxies which have been discovered by various techniques. One such group of interesting galaxies are those listed by Markarian $(1967,1969 a, b)$ and Markarian and Lipovetsky (1971, 1972). These objects were discovered using objective-prism Schmidt plates and have the characteristic that they radiate excessively in the ultraviolet compared with normal galaxies. Since their discovery, however, a large quantity of spectral and photometric data (see, e.g., Weedman 1973) has led to the recognition that in fact the Markarian galaxies form a physically heterogeneous collection which includes galaxies of different morphological types with different emission characteristics.

It is clearly appropriate to observe a number of the galaxies over a wide range of wavelengths and, by necessity, with varying spectral resolutions. An observational program of a selected list of 18 Markarian galaxies which involves three kinds of measurements has therefore been carried out at the Hale $5 \mathrm{~m}$ telescope over the period 1970-1975.

1. Slit spectra were obtained to study relative emission-line intensities, to study the spatial distribution of emission in the galaxy, and to study line profiles. Spectra of a large number of Markarian galaxies have already been published. A review of the work preceding 1972 is given by Markarian (1972); spectra of several selected Markarian galaxies have been published more recently (see, e.g., Khachikian and Weedman 1974).

2. The multichannel spectrometer was used to obtain absolute spectral energy distributions for the galaxies from 3300 to $10,000 \AA$.

3. Broad-band infrared observations were made at 1.65 and $2.2 \mu$ and, in half the cases, at 3.5 and $10 \mu$.
Infrared data on seven of these Markarian galaxies are included in the study of extragalactic objects by Rieke and Low (1972). Stein and Weedman (1975) have observed five of the program galaxies at $3.5 \mu$ in their study of Seyfert galaxies.

The list of Markarian galaxies observed in the present program is given in Table 1. All were taken from the first two lists of Markarian $(1967,1969 a)$, and all were known to have emission lines.

\section{THE OBSERVATIONS}

The slit spectra were all obtained with the Cassegrain image-tube spectrograph. Several spectra of each object were obtained, some covering different spectral ranges, and others simply duplicating observations. Normally, the galaxies were held fixed on the slit. The dispersion varied from $30 \AA \mathrm{mm}^{-1}$ to $190 \AA \mathrm{mm}^{-1}$, and the resolution was typically 2-10 ̊. Dr. W. L. W. Sargent provided spectra of Markarian objects 79 and 124, and Dr. M. Schmidt obtained a spectrum of Markarian 54.

The multichannel spectrometer at the Hale telescope was used to obtain absolute spectral energy distributions of all the galaxies. Observations were made with bandpasses of $40 \AA$ for $\lambda 3300$ to $\lambda 5800$ and $80 \AA$ for $\lambda 5700$ to $\lambda 10,000$ in most cases. Other observations with bandpasses of 80 and $160 \AA$ were also sometimes available. The entrance apertures were usually $7^{\prime \prime}, 10^{\prime \prime}$, or $14^{\prime \prime}$, but some observations were also made with a $5^{\prime \prime}$ aperture if conditions were ideal. Observations were reduced by usual procedures, and absolute fluxes are based on the calibrations of Vega given by Oke and Schild (1970).

The infrared observations at $1.65 \mu(1.5-1.8 \mu)$, $2.2 \mu(2.0-2.4 \mu)$, and $3.5 \mu(3.2-3.8 \mu)$ were obtained at the $\mathrm{f} / 16$ Cassegrain focus of the Hale telescope with a conventional infrared photometer. The $10 \mu(8-13 \mu)$ measurements were obtained with the $\mathrm{f} / 72$ wobbling 
TABLE 1

Program Objects, Selected Fluxes, and Luminosities

\begin{tabular}{|c|c|c|c|c|c|c|c|c|c|c|c|c|c|c|c|c|c|}
\hline \multirow[b]{2}{*}{$z \S$} & \multirow[b]{2}{*}{ bII } & \multicolumn{5}{|c|}{$\left(\right.$ ergs $\left.\mathrm{cm}^{-2} \mathrm{~s}_{\mathrm{v}}^{-1} \mathrm{~Hz}^{-1}\right)$} & \multicolumn{3}{|c|}{$\begin{array}{c}j \times 10^{15} \\
\left(\operatorname{ergs~} \mathrm{cm}^{-2} \mathrm{~s}^{-1}\right)\end{array}$} & \multicolumn{5}{|c|}{$\underset{\left(\operatorname{ergs} s^{-1}{ }_{\mathrm{Hz}}^{-1}\right)}{\log \mathrm{F}_{\nu}}$} & \multicolumn{3}{|c|}{$\begin{array}{l}\mathrm{Jx} 10^{-40} \\
\left(\operatorname{ergs~\mathrm {s}^{-1}}\right)\end{array}$} \\
\hline & & $\begin{array}{l}V^{*} \\
7 "\end{array}$ & $\begin{array}{l}V^{*} \\
10 "\end{array}$ & $2.2 \mu$ & $3.5 \mu$ & $\begin{array}{l}10 \mu \\
\leq 4 "\end{array}$ & $\underset{7 "}{\mathrm{H} B}$, & $\begin{array}{l}\mathrm{H} \beta, \\
10^{\prime \prime}\end{array}$ & $\begin{array}{l}\mathrm{H} \beta, \\
14^{\prime \prime}\end{array}$ & $\begin{array}{l}\mathrm{V} \\
71 "\end{array}$ & $\begin{array}{c}\mathrm{V} \\
10^{\prime \prime}\end{array}$ & $2.2 \mu$ & $3.5 \mu$ & $\begin{array}{l}10 \mu \\
\leq 4 "\end{array}$ & $\begin{array}{c}\mathrm{HB}, \\
7 "\end{array}$ & $\begin{array}{c}\mathrm{HB}, \\
10^{\prime \prime}\end{array}$ & $\begin{array}{l}\mathrm{HB}, \\
14 "\end{array}$ \\
\hline $1 \ldots 0.0160$ & -30 & -25.76 & -25.63 & -25.09 & -25.11 & -24.08 & 69 & 58 & 77 & 28.27 & 28.40 & 28.94 & 28.92 & 29.95 & 7.6 & 6.4 & 8.4 \\
\hline $3 \ldots 0.0137$ & +22 & -25.20 & -25.04 & -24.29 & -24.49 & -23.39 & 311 & 368 & & 28.70 & 28.86 & 29.61 & 29.41 & 30.51 & 25.0 & 29.6 & \\
\hline $34 \ldots 0.0510$ & +50 & -25.75 & & -25.00 & -24.91 & -24.51 & 72 & & & 29.28 & & 30.02 & 30.12 & 30.52 & 80 & & \\
\hline $6 \ldots 0.0197$ & +26 & -25.27 & $\left\{\begin{array}{l}-25.10 \\
-25.34\end{array}\right.$ & -24.29 & -24.13 & -23.76 & 479 & 598 & & 28.94 & $\left\{\begin{array}{l}29.11 \\
28.87\end{array}\right.$ & 29.92 & 30.08 & 30.45 & 80 & 99 & \\
\hline $9 \ldots 0.0401$ & +29 & -25.50 & -25.44 & -24.48 & -24.13 & -23.84 & 189 & 222 & & 29.32 & $\begin{array}{l}128.81 \\
29.38\end{array}$ & 30.34 & 30.69 & 30.98 & 130 & 153 & \\
\hline $10 \ldots 0.0293$ & +30 & & -25.44 & -24.74 & -24.75 & $<-23.89$ & & 199 & & & 29.11 & 29.81 & 29.80 & $<30.66$ & & 73 & \\
\hline $79 \ldots 0.0220$ & +29 & & -25.21 & -24.37 & -24.16 & -23.84 & & 531 & & & 29.10 & 29.94 & 30.15 & 30.47 & & 110 & \\
\hline $124 \ldots 0.0566$ & +48 & -25.68 & & -24.70 & -24.39 & -24.40 & 101 & & & 29.43 & & 30.41 & 30.72 & 30.71 & 138 & & \\
\hline $13 \ldots 0.0052$ & +31 & -25.97 & & -25.52 & & & 24 & & & 27.09 & & 27.54 & & & 0.28 & & \\
\hline $19 \ldots 0.0141$ & +41 & -26.00 & & -25.61 & & & $33 ! 5$ & 5") & & 27.92 & & 28.31 & & & 2.80 & & \\
\hline $33 . \ldots 0.0054$ & +53 & & -25.09 & & & & & 434 & 412 & & 28.00 & & & & & 5.4 & 5.1 \\
\hline $35 \ldots 0.0034$ & +53 & -25.50 & & -25.15 & & & 333 & & & 27.19 & & 27.54 & & & 1.65 & & \\
\hline $36 \ldots 0.0022$ & +67 & -26.09 & -25.97 & -26.14 & & & 109 & 146 & & 26.22 & 26.34 & 26.18 & & & 0.23 & 0.30 & \\
\hline $49 \ldots 0.0042$ & +65 & & -25.85 & -25.89 & & & & 134 & & & 27.03 & 26.99 & & & & 1.01 & \\
\hline $52 \ldots 0.0062$ & +64 & -25.32 & & -24.75 & & -23.97 & 187 & & & 27.89 & & 28.46 & & 29.24 & 3.08 & & \\
\hline $54 \ldots 0.045$ & +85 & & -25.60 & -25.50 & & & & 29 & & & 29.32 & 29.42 & & & & 25.1 & \\
\hline $59 \ldots 0.0027$ & +82 & -25.49 & & -25.50 & & $<-24.62$ & 719 & & 993 & 27.00 & & 26.99 & & $<27.87$ & 2.24 & & 3.10 \\
\hline $67 \ldots 0.0036$ & +77 & -26.23 & -25.77 & -26.10 & & -24.7 & 39 & & & 26.51 & 26.97 & 26.64 & & 28.0 & 0.22 & & \\
\hline
\end{tabular}

\footnotetext{
* Measured at $\log V=14.74-\log \mathrm{z}, \mathrm{H}=50 \mathrm{~km} \mathrm{~s}{ }^{-1} \mathrm{Mpc}^{-1}$

+ Aperture range from 7-14" as indicated in figures 5-8.

$\S 2$ from Weedman and Khachikian $(1968,1969)$ and Sargent (1972).
}

secondary photometer of the Hale telescope; calibrations were made using standard stars; the flux levels at the effective wavelengths are given in Wilson et al. (1972). The 2.2 and $3.5 \mu$ measurements were generally obtained with apertures ranging from 7.5 to $15^{\prime \prime}$; in some cases, multiaperture data were obtained of the same object. The $10 \mu$ measurements covered only the central $2^{\prime \prime}$ or $3^{\prime \prime}$ of the galaxies. Most of the 10 and $3.5 \mu$ measurements were obtained on one night only. For MKN 3 a single measurement of the $20 \mu$ flux is also given.

All the spectroscopic and photometric data were obtained from 1970 December through 1975 February. With the possible exception of MKN 6, the optical and infrared data showed no evidence of significant variability but the coverage was inadequate to allow a quantitative discussion about variability or nonvariability.

\section{RESULTS}

\section{a) Continuum Energy Distribution and Emission Line Intensities}

The absolute spectral energy distributions between 3300 and $10,000 \AA$, i.e., $\log v(\mathrm{~Hz})=14.50$ to 14.95 , are shown in Figures 1 to 4 ; the groupings are discussed below. In the case of MKN 6, the visual flux in a $10^{\prime \prime}$ aperture on 1969 October was 0.6 mag greater than that on 1972 February. The data of 1972 February are plotted in Figures 1 and 5.
Since the photoelectric spectral energy distributions cover a wide wavelength range, it is relatively straightforward to draw in a continuum. This was done by requiring that it be smooth except in the region of the $\mathrm{H}$ and $\mathrm{K}$ lines of $\mathrm{Ca}$ II where a rather abrupt drop in intensity is observed if the radiation comes largely from stars. Except for these lines, features were assumed to be largely emission rather than absorption. Continua observed with different entrance apertures tended to look almost identical except for differences in the absolute flux levels.

Isolated emission lines, such as the [O II] lines $\lambda \lambda 3726,3729$, can be readily measured relative to the adopted continuum from the spectrophotometric data. In the case of crowded or blended emission lines, the spectrophotometric observations can give only the equivalent width of the blended feature. To separate the individual lines, microphotometer tracings of the slit spectra were used along with information about known line ratios, e.g., the ratio of the $\lambda \lambda 5007$ to 4959 lines of [O III]. Some blended features, particularly in the broad emission-line objects, could not be separated adequately even with the slit spectra.

The emission-line equivalent widths were converted to intensities by using the absolute fluxes for the adopted continuum. The line intensities relative to $\mathrm{H} \beta$ are listed in Tables 2 and 3 , while the absolute intensities are given in Table 1 . The line intensities inferred from observations with different angular apertures agreed well with each other in the case of 


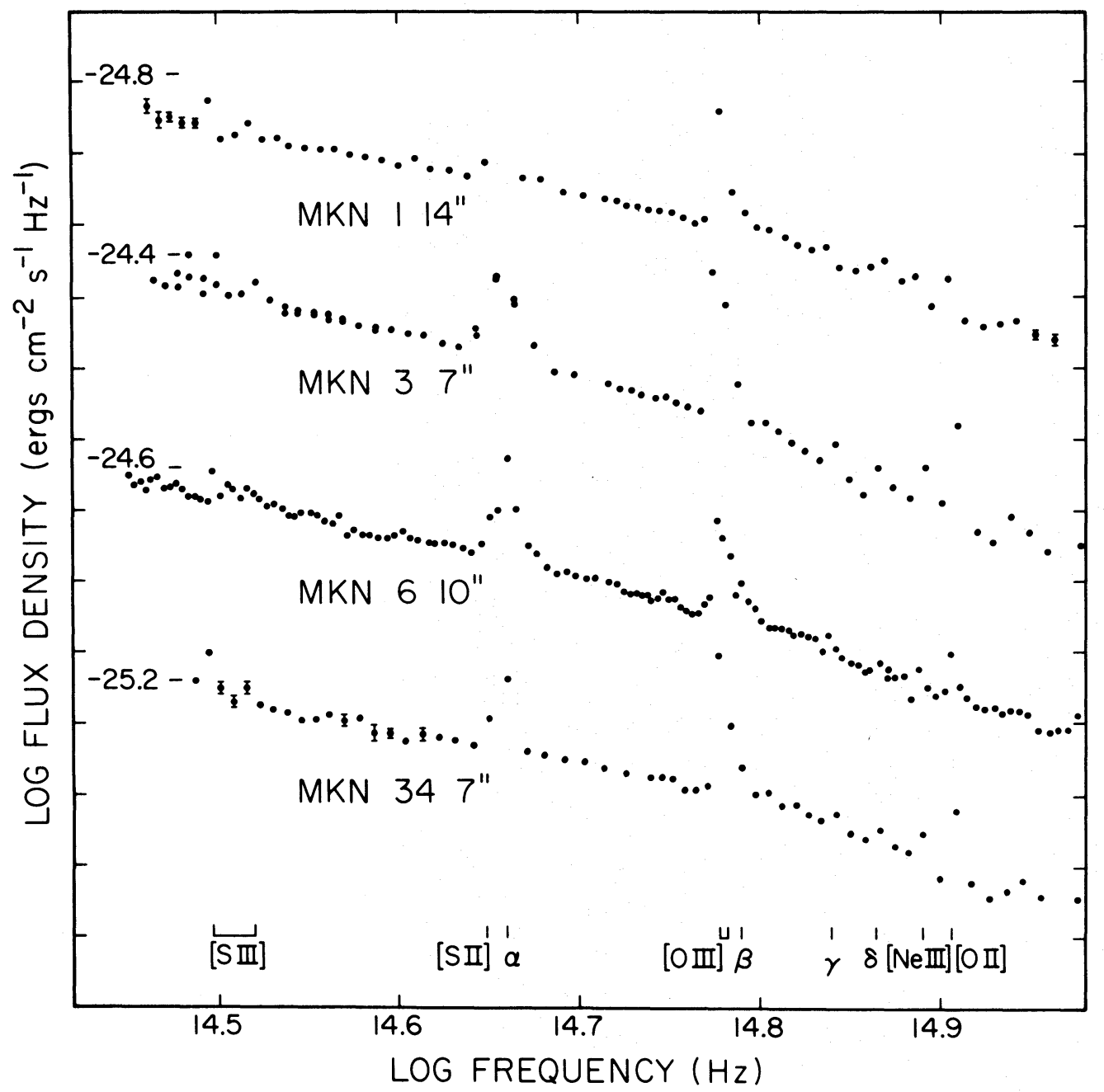

Fig. 1.-Absolute spectral energy distributions. All plots have been shifted to correspond to rest frequencies; no reddening correction has been applied. Standard deviations are shown if they are larger than 5 percent. The ordinate is graduated in intervals of 0.4 in log flux density. An absolute value for each scan is indicated. Positions of the stronger emission lines are marked.

Seyfert like objects (see below), indicating that the emission in these cases comes from an object with a diameter of 7 " or less. Indications of the breadths of the permitted and forbidden lines are also given in Tables 2 and 3 ; the presence or absence of $\mathrm{Fe}$ II is also indicated.

The continuum and infrared data are presented in Figures 5 through 8; selected data are incorporated in Table 1. In those cases where measurements of Rieke and Low (1972) were duplicated (MKN 1, $9,10,34,52$, and 79) the results were in agreement except for MKN 52; in this case Rieke and Low measured a $10 \mu$ flux of $0.24 \pm 0.04 \times 10^{-23}$ ergs $\mathrm{s}^{-1} \mathrm{~cm}^{-2} \mathrm{~Hz}^{-1}$ in a $6^{\prime \prime}$ aperture in contrast to the value $0.10 \pm 0.02 \times 10^{-23} \mathrm{ergs} \mathrm{s}^{-1} \mathrm{~cm}^{-2} \mathrm{~Hz}^{-1}$ measured in a $2^{\prime \prime}$ aperture. The $10 \mu$ flux of MKN 33 is taken from Rieke and Low (1972). The 1.6, 2.2, and $3.5 \mu$ fluxes of MKN 6 may have shown a dip by $\sim 0.5 \mathrm{mag}$ in 1971 October relative to the data of 1971 March and 1972 January; this is uncertain be- cause of marginal conditions during 1971 October. The 1972 January data are plotted in Figure 5.

\section{b) Nature of Markarian Galaxies}

An examination of the spectral data listed in Tables 2 and 3 shows that it is possible to divide the sample Markarian galaxies into those containing broad emission lines and those whose emission features are all sharp. The former group will be identified below with Seyfert galaxies while the latter group will be identified with extragalactic $\mathrm{H}$ II regions.

\section{SEYFERT GALAXIES}

a) Phenomenology $(M K N 1,3,6,9,10$, $34,79,124)$

The sample of galaxies with broad emission lines shows the same properties, and range in properties, as do the classical Seyfert galaxies described by Anderson (1970). All have prominent semistellar nuclei, and 


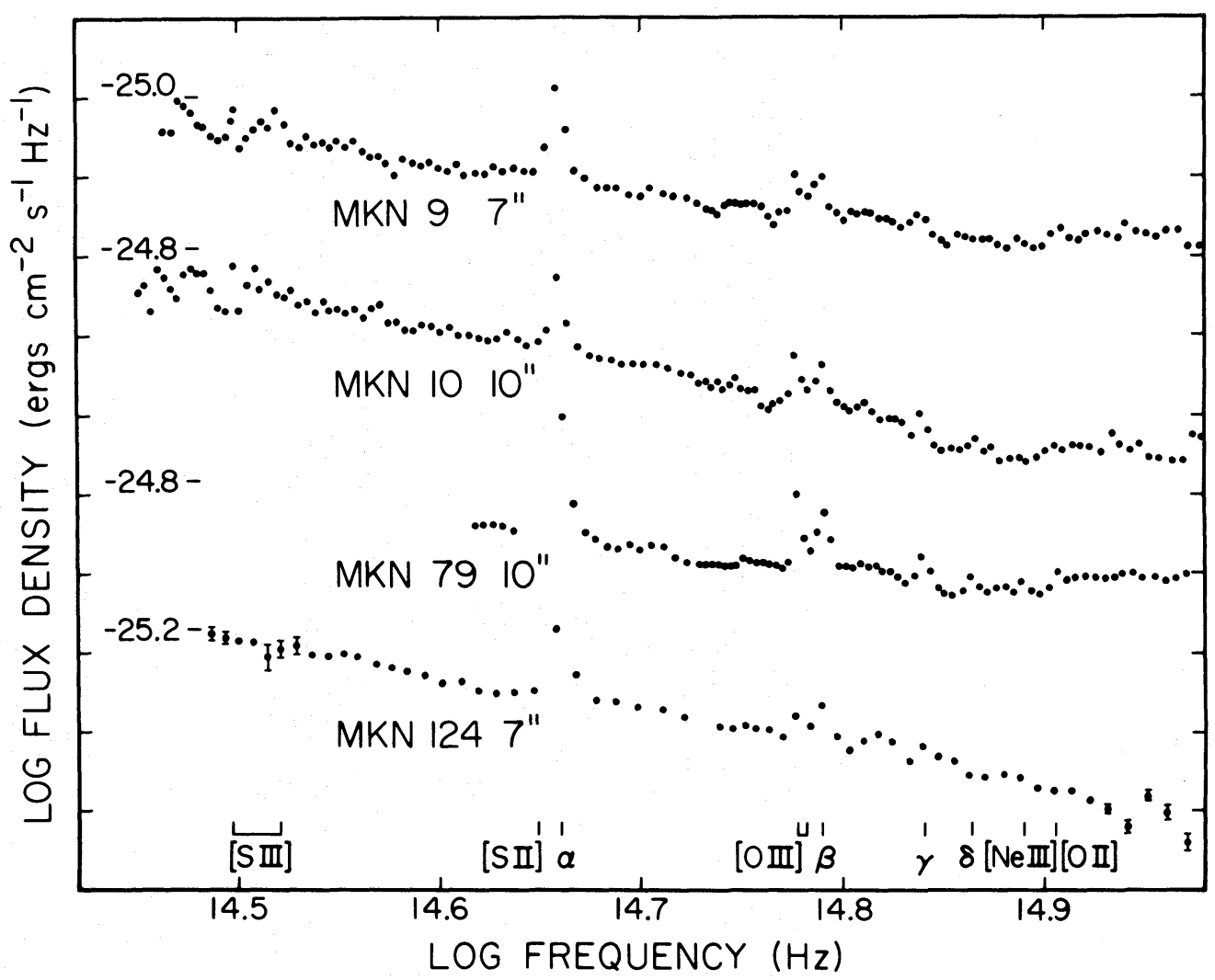

Fig. 2.-Same as Fig. 1

the spectra all show [Ne v] and often [O $\mathrm{I}]$. The forbidden lines are all appreciably widened (full width at half-intensity $\sim 700 \mathrm{~km} \mathrm{~s}^{-1}$ ); and often, but not always, the permitted lines are very much wider than the forbidden ones. In addition, the relative line intensities, continuous energy distributions, line strengths relative to the continuum, and absolute luminosity are also characteristic of the Seyfert galaxies. The galaxies have been previously identified as Seyfert galaxies by Weedman (1970) [MKN 1, 3, and 6], Arp et al. (1968) [MKN 9 and 10], Arakelian, Dibay, and Yesipov (1970) [MKN 79], Weedman and Khachikian (1968) [MKN 34], and Sargent (1972) [MKN 124].

Reddening.-From Table 2 it is seen that the observed ratio of the intensities of $\mathrm{H} \alpha$ to $\mathrm{H} \beta$ range from 3.1 to 5.2. As indicated in Table 2, in some cases these ratios are uncertain since the [N II] and [S II] lines are not always resolved from $\mathrm{H} \alpha$ in the data. These ratios should be compared with the radiative recombination ratio which varies from 2.7 for $T_{e}=20,000 \mathrm{~K}$ to 2.95 for $T_{e}=6,000 \mathrm{~K}$. The observed results for some of these objects therefore suggest either that the Balmer lines are not formed entirely by recombination or that there is interstellar reddening within the Galaxy or in the Markarian galaxy. Osterbrock and Parker (1965) suggested a mechanism by which the large $\mathrm{H} \alpha / \mathrm{H} \beta$ ratio found in NGC 1068 could be explained without reddening. Wampler (1971), on the other hand, using [S II] lines, has demonstrated that in the Seyfert galaxies NGC 1068 and NGC 1275 there is substantial reddening and that the true ratio of $\mathrm{H} \alpha / \mathrm{H} \beta$ is close to the recombination value. Shields, Oke, and Sargent (1972) found for 3C 120 that the various line ratios were at least consistent with reddening and a recombination ratio for $\mathrm{H} \alpha / \mathrm{H} \beta$, although Shields (1974) showed that collisional excitation in his model could produce an intrinsic line ratio as high as 3.5.

Since the present observations of Markarian galaxies provide only $\mathrm{H} \alpha: \mathrm{H} \beta: \mathrm{H} \gamma$ ratios at best and since the correct ratios may not be the recombination values, no reddening corrections have been applied to the data presented in Tables 1 and 2 and Figures 1, 2, 5, and 6. In any case, the observed $\mathrm{H} \alpha / \mathrm{H} \beta$ ratios show that reddening, if present, cannot be large.

Emission-line ratios. - Khachikian and Weedman $(1971 a, 1974)$ have discussed the separation of Seyfert galaxies into two classes. One of these (class 1) contains the majority of Seyferts, and has permitted lines with a component which is much broader than the forbidden lines while in the second class the permitted and forbidden lines are of comparable width. NGC 4151 and $3 \mathrm{C} 120$ could be considered prototypes of the former class while NGC 1068 typifies the latter. On the basis of this classification, MKN 9, 10,79, and 124 belong to class 1 while MKN 1,3 , and 34 belong to class 2 . Markarian 6 has been classified by Khachikian and Weedman (1974) as belonging to class 


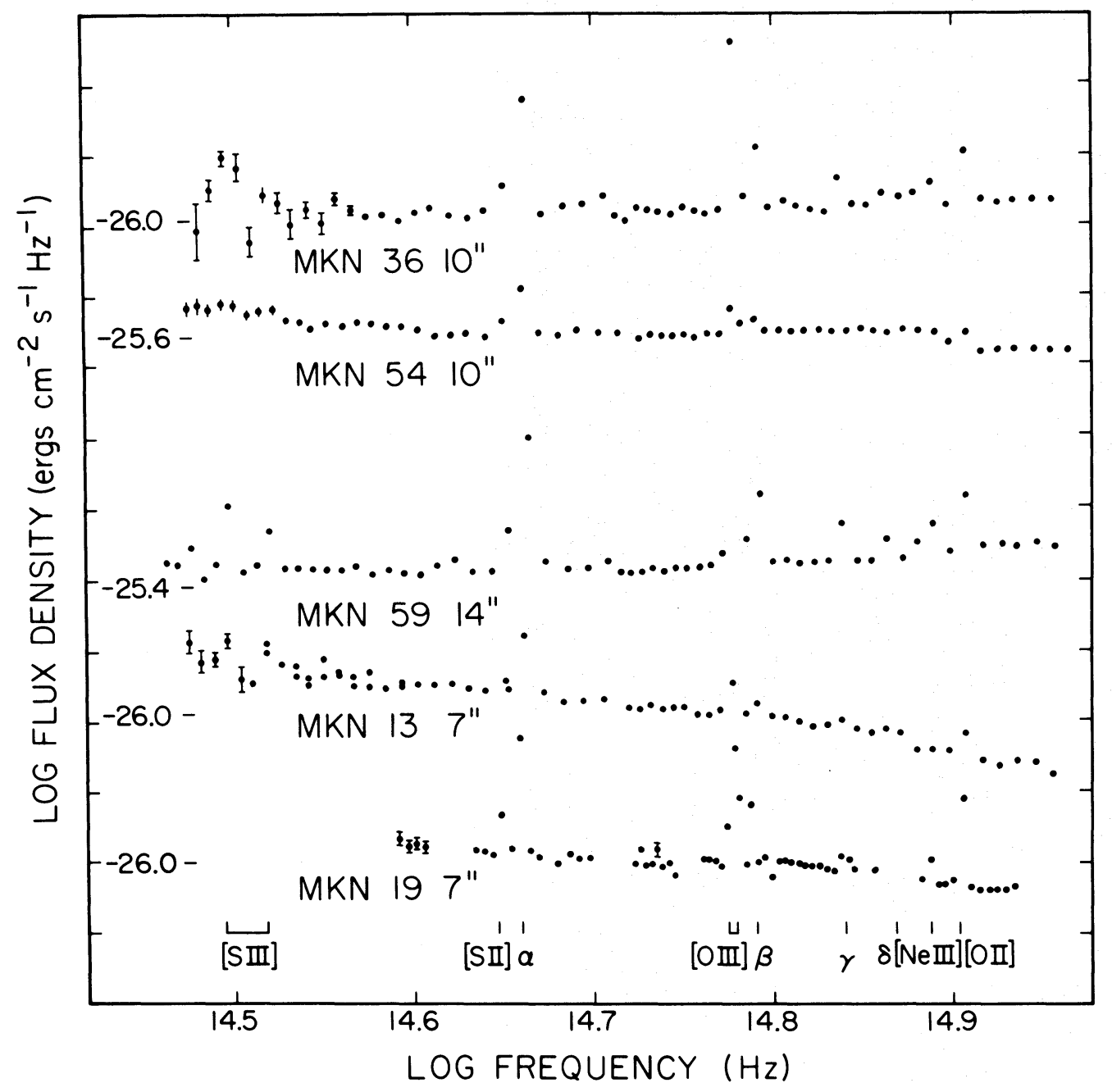

FIg. 3.-Same as Fig. 1 


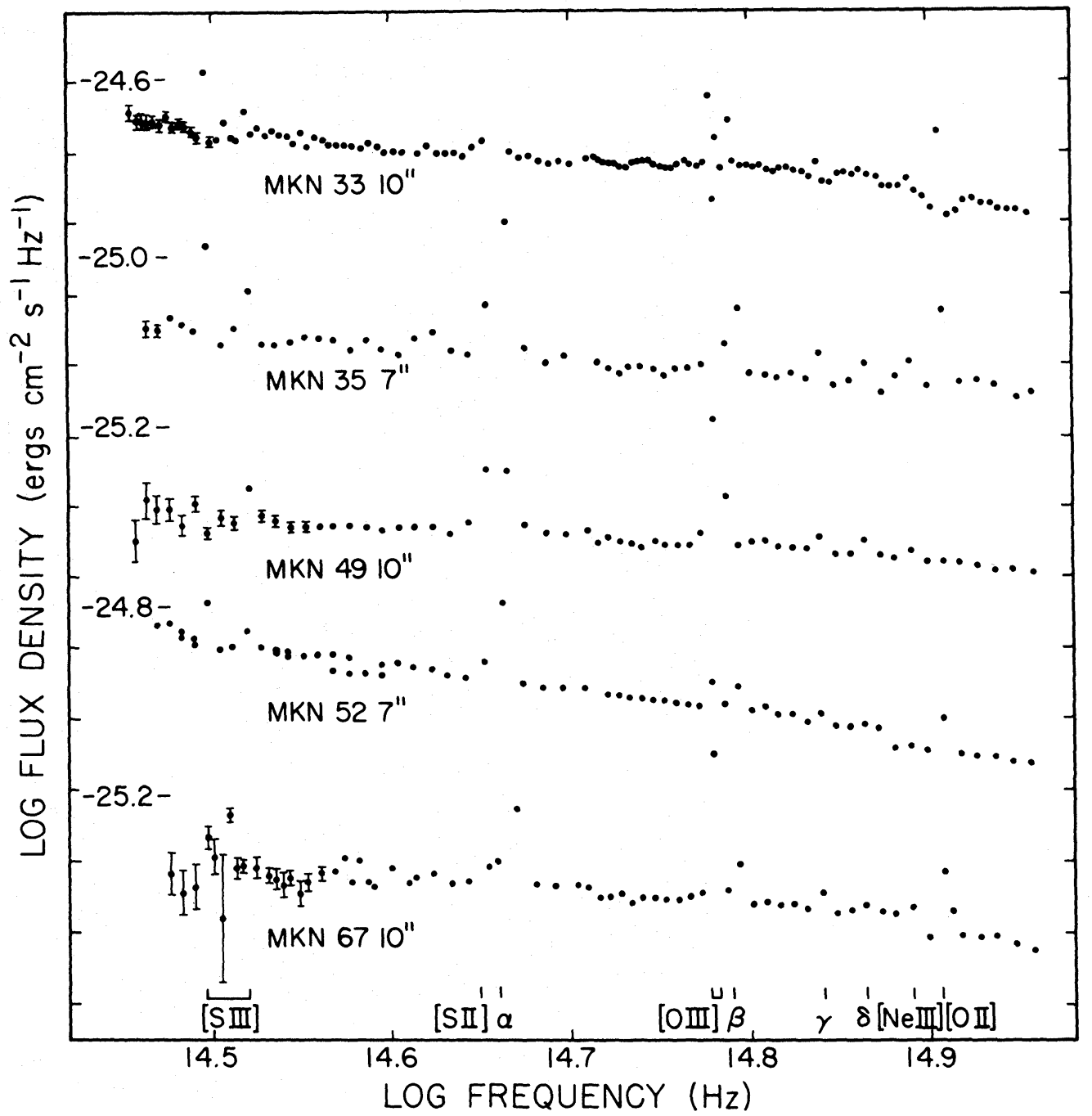

FIG. 4.-Same as Fig. 1. In MKN 33 one data point is missing in the H $\alpha$ emission line. In MKN 67 the resolution was the same as for MKN 33 but alternate points were not measured. 


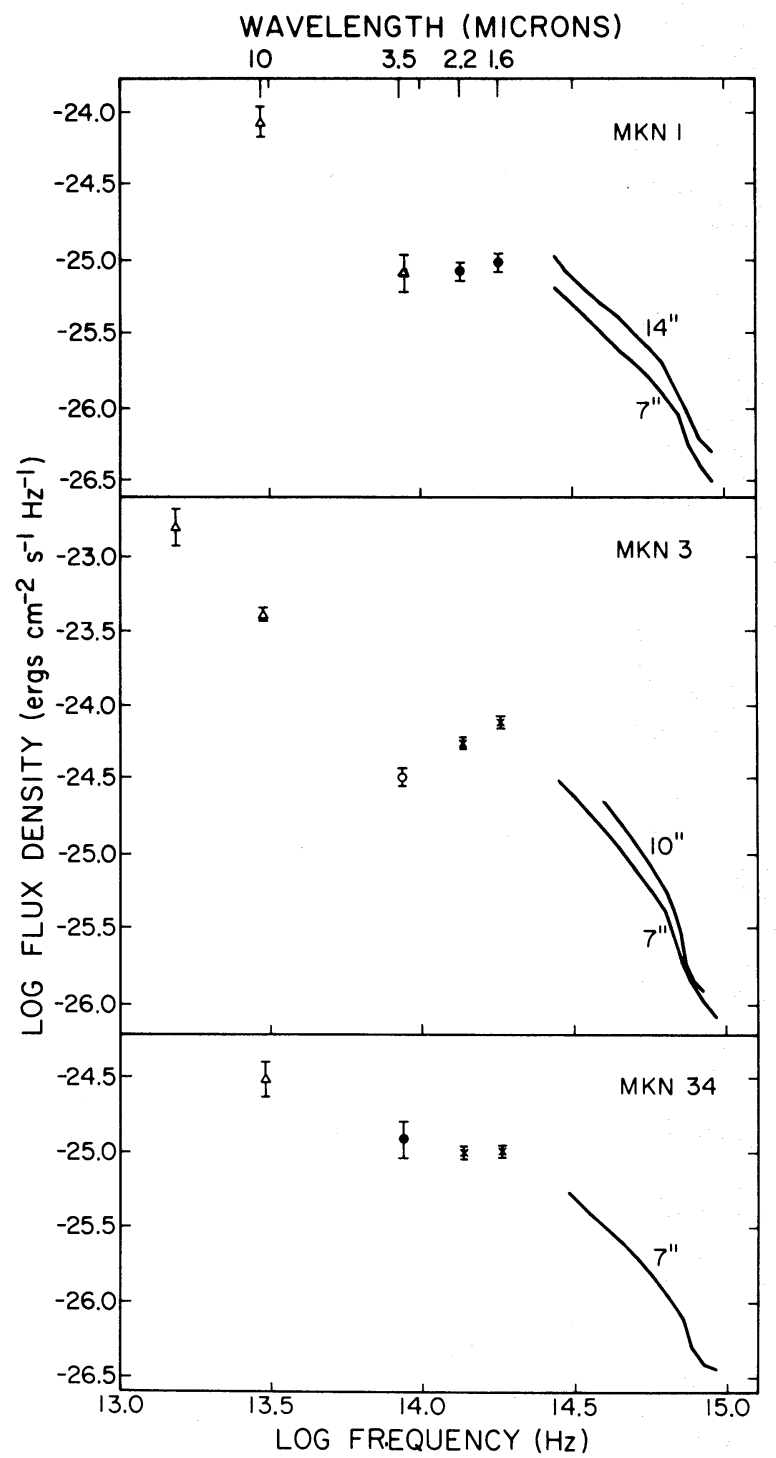

Fig. 5.-Continuum energy distributions from 0.33 to $20 \mu$. The solid curves represent the multichannel observations; the diameter of the circular aperture used is given in arc seconds. In the case of broad-band infrared measures standard deviations are shown or in case of upper limits, $3 \sigma$ limits. Aperture diameters are given by the following symbols: $\triangle, \leq 5^{\prime \prime} ; 0,7^{\prime \prime} ; \times, 10^{\prime \prime} ; \bigcirc$, averages $7^{\prime \prime}-15^{\prime \prime} ;+, 15^{\prime \prime}$. Measurements by Rieke and Low (1972) with a 6" aperture are marked $R$.

2 on the basis of slit spectra. The spectrophotometric results (see Fig. 1) show the existence of a very broad component for the permitted lines such as $\mathrm{H} \beta$, however, and on this basis we have classified MKN 6 as class 1 .

As remarked by Khachikian and Weedman (1974) the Seyfert galaxies of class 2 generally show a high [O III] $/ \mathrm{H} \beta$ ratio; of the sample observed, $\mathrm{MKN} 1$, 3 , and 34 have a ratio $[\mathrm{O} \mathrm{III}] / \mathrm{H} \beta$ in the range $12-13$. In contrast the ratios for the four class 1 Seyfert galaxies MKN 9, 10, 79, and 124 are all close to 0.9

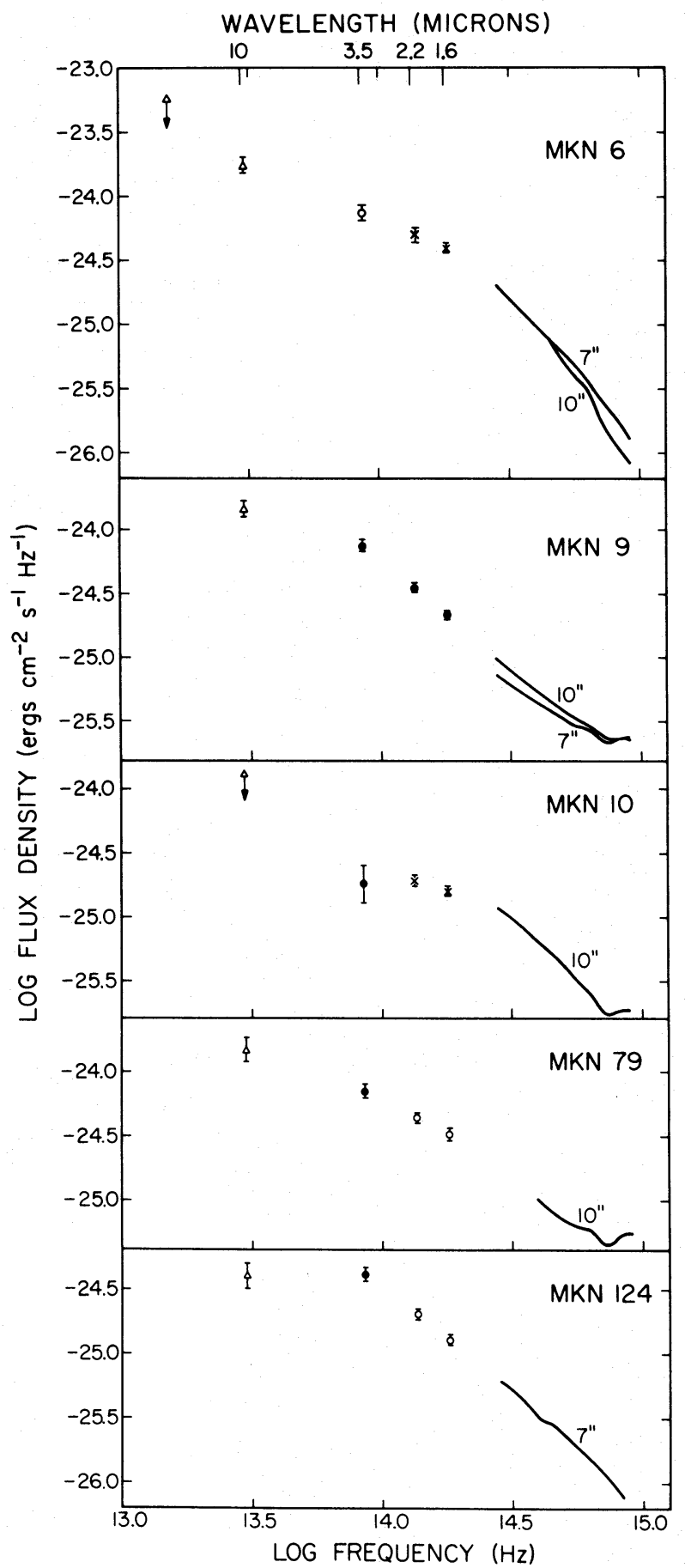

FIG. 6.-Same as Fig. 5

while the ratio for $\mathrm{MKN} 6$ is 1.65 . The ratio of [O III] to most other forbidden lines such as [S III], [S II], [N II], [Ne III], and [O II] is approximately constant in all the Seyferts.

A comparison of the relative emission-line intensities in the class 2 Seyferts MKN 1, 3, and 34 shows a remarkable similarity among all three objects. 


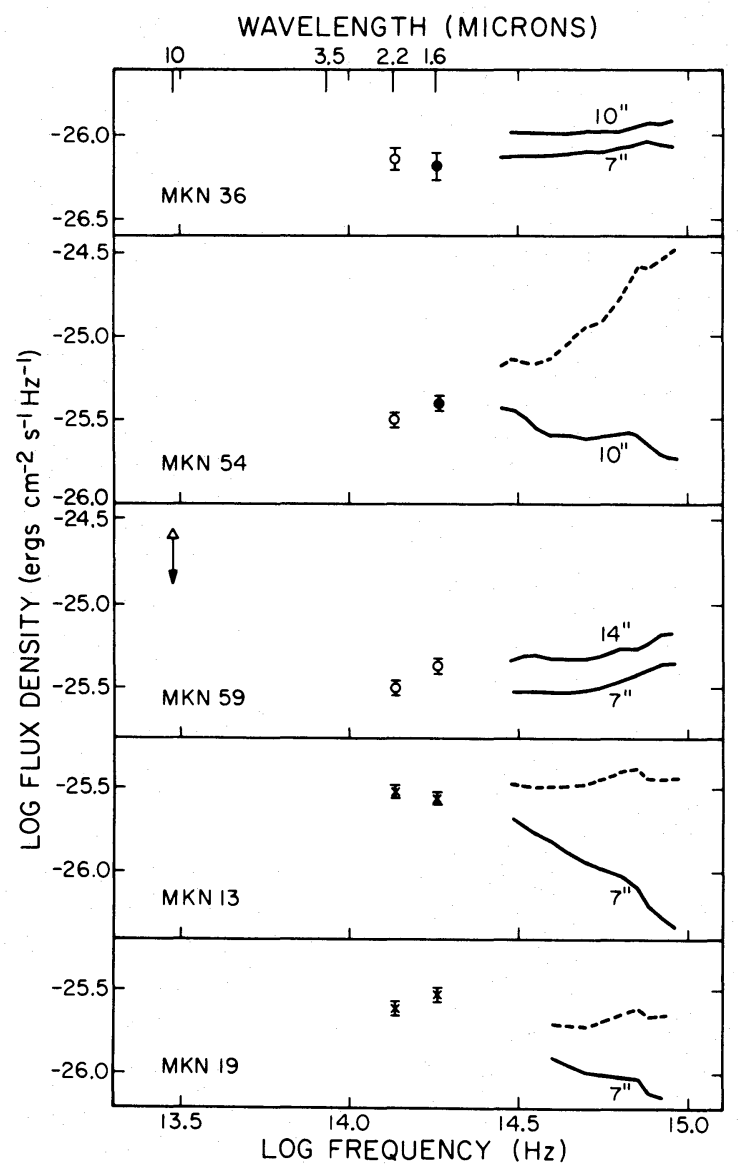

FIG. 7.-Continuum energy distributions as in Fig. 5. The broken curves represent the same observations with reddening corrections applied as described in the text.

The ratio of the two [Ar IV] lines $\lambda \lambda 4711$ and 4740 indicate an electron density of $N_{e} \approx 10^{5} \mathrm{~cm}^{-3}$. The ratio of the [O III] lines $(\lambda 4959+\lambda 5007 / \lambda 4363$ is 100$)$; for an electron temperature in the neighborhood of $12,000 \mathrm{~K}$ this ratio also implies $N_{e} \approx 10^{5} \mathrm{~cm}^{-3}$. On the other hand, in these three objects the strength of the [O II] lines $\lambda \lambda 3727,3729$ are stronger than the [O II] lines at $\lambda \lambda 7320,7330$, even without any reddening corrections. For an electron temperature in the neighborhood of 10,000 to $20,000 \mathrm{~K}$, this implies $N_{e}<10^{3} \mathrm{~cm}^{-3}$. In a general way the arguments of Osterbrock and Parker (1965) and Shields and Oke (1975) about NGC 1068 apply to MKN 1, 3, and 34, although NGC 1068 has relatively stronger lines of highly ionized ions such as [Ne v] and weaker lines of low-ionization ions such as [O I], [O II], and [S II]. In summary, in MKN 1, 3, and 34 there are at least two density domains, one with $N_{e} \approx 10^{5} \mathrm{~cm}^{-3}$ and one with $N_{e}<10^{3} \mathrm{~cm}^{-3}$.

In the case of the class 1 Markarian galaxies 6, $9,10,79$, and 124 , the inferred densities are quite different. In order to suppress the [O III] $\lambda \lambda 4959$, 5007 lines relative to $H \beta$ in the broad line region, $N_{e}$ must be greater than $10^{8} \mathrm{~cm}^{-3}$. In addition, per-

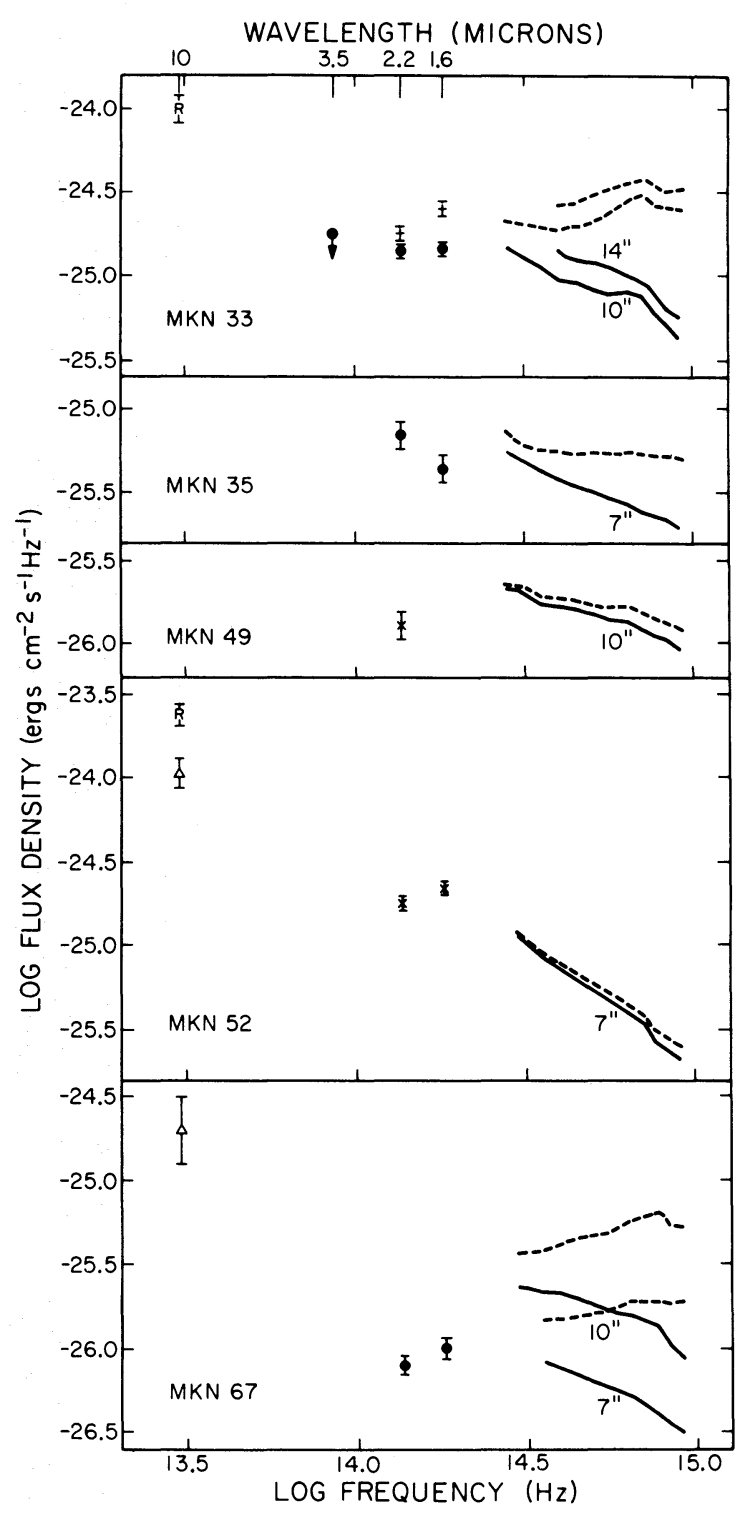

Fig. 8. - Same as Fig. 7

mitted lines of $\mathrm{Fe}$ II are definitely present in MKN 9, 10,79 , and 124, and may be present in MKN 6; Boksenberg et al. (1975) have listed Fe II as being present in MKN 6. Densities as high as $10^{8} \mathrm{~cm}^{-3}$ are consistent with the appearance of Fe II lines (Wampler and Oke 1967; Bahcall and Kozlovsky 1969). In addition to the high densities found above, there is evidence for at least two other density regions in the class 1 Seyferts. The [O III] ratio $(\lambda \lambda 4959+5007) /$ $\lambda 4363 \approx 10$; for temperatures in the range $10,000-$ $20,000 \mathrm{~K}$ this requires $N_{e} \approx 10^{6} \mathrm{~cm}^{-3}$. At the same time [O II] line ratio $(\lambda \lambda 3727+3729) /(\lambda \lambda 7320+$ $7330) \approx 3$ implies $N_{e} \leq 10^{3} \mathrm{~cm}^{-3}$. For this group of objects there thus appear to be three domains where $N_{e} \geq 10^{8}, N_{e} \approx 10^{6}$, and $N_{e} \leq 10^{3} \mathrm{~cm}^{-3}$.

The ionization processes are also different in the class 1 Seyferts from those in class 2 objects, as is 
shown by the much higher ratio of the [Ne v] $\lambda 3345$ line intensity to the [O III] $\lambda 5007$ line intensity in class 1 as compared to class 2 objects. The He II $\lambda 4686 / \mathrm{He}$ I $\lambda 5876$ ratio appears to be larger in MKN 1,3 , and 34 than in MKN 9, 10, and 79.

Finally, there is evidence that in some objects, notably MKN 1 and MKN 3, the forbidden emission lines arise in a region that is a sizable fraction of the entire galaxy, and not just from the bright nucleus. These galaxies have diameters of $6 \mathrm{kpc}$, and emission is present as far out as $1.5 \mathrm{kpc}$ from the nucleus. Furthermore, this emission spectrum is the same as the nuclear emission line spectrum; [O III] $/ \mathrm{H} \beta \approx 12$ and $\left[\mathrm{O}_{\mathrm{I}}\right]$ and $[\mathrm{Ne} \mathrm{v}]$ are simultaneously present.

Continua.-The continua of the Seyfert galaxies as obtained from the multichannel spectrometer and the infrared photometry are presented in Figures 5 and 6. It is seen that nearly all the Seyferts are marked by having large $10 \mu$ fluxes. In MKN 6, 9, and 79 the continuum rises smoothly from the visible into the infrared while an inflection is obviously present at $\sim 3 \mu$ in MKN 3 and, less pronounced, in MKN 1 and 34. In MKN 9, 10, and 79 there is a definite increase in the continuum at the ultraviolet end of the observations; an upward inflection in the continua of MKN 1, 3, and 34 is also present. In the latter three, there is a break at $\sim 3900 \AA$ corresponding to the $H$ and $\mathrm{K}$ lines, a break which is absent in the remaining Seyfert galaxies studied except perhaps in MKN 6 . In addition, it is seen that there is a correlation between the overall appearance of the continuum energy distribution and the separation into Khachikian/ Weedman classes.

It has become fashionable to decompose the continuum spectrum of Seyfert galaxies into a spectrum representing the galactic stellar component plus an additional component presumably arising in the nucleus (see, e.g., Khachikian and Weedman 1974; Penston et al. 1974). This decomposition has been illustrated in Figures 9 and 10 where a spectrum of M31 (Sandage, Becklin, and Neugebauer 1969) is shown combined with nonstellar contributions which correspond to emission with $f_{v} \propto v^{-1.7}$ (Fig. 9) and blackbody radiation from a source at $200 \mathrm{~K}$ (Fig. 10). The slope of -1.7 adopted for the power-law spectrum has been taken as representative of MKN 9, 79, and 6. It equals the steep spectral slopes shown in many quasi-stellar sources at $\lambda \approx 2 \mu$ (Oke, Neugebauer,

TABLE 2

Observed Line INTENSITIES Reduced to $H \beta \equiv 1$

\begin{tabular}{|c|c|c|c|c|c|c|c|c|c|}
\hline$\lambda$ & Ion & & Ty $\mathrm{pe}$ & 2 & & & y pe & 1 & \\
\hline Markarian & Number & 1 & 3 & 34 & 6 & 9 & 10 & 79 & 124 \\
\hline 9545 & P8 [ [ I III] & 2.68 & 1.03 & 2.94 & 0.23 & 0.14 & - & - & nd \\
\hline 9069 & {$\left[\begin{array}{ll}S & I I I\end{array}\right]$} & 1.20 & 1.16 & 1.24 & 0.10 & 0.34 & nd & - & nd \\
\hline 7319,30 & {$\left[\begin{array}{ll}0 & \mathrm{II}\end{array}\right]$} & 0.76 & nd & nd & 0.05 & 0.08 & nd & - & $\mathrm{p}$ \\
\hline 7136 & {$\left[\begin{array}{ll}A & I I I\end{array}\right]$} & nd & nd & nd & nd & nd & nd & nd & nd \\
\hline 6717,31 & {$\left[\begin{array}{ll}S & I I\end{array}\right]$} & 1.52 & 1.81 & 1.68 & 0.26 & & 0.10 & - & $0.31=$ \\
\hline 6548,83 & {$\left[\begin{array}{ll}N & I I\end{array}\right]$} & 3.88 & 6.43 & 2.38 & 0.39 & 4.14 & \multirow{2}{*}{\} 3.33} & \multirow{2}{*}{3.11} & \multirow{2}{*}{\} 4.95} \\
\hline 6562 & $\mathrm{H} \alpha$ & 4.27 & 3.87 & 3.58 & 5.15 & & & & \\
\hline 6300,63 & {$\left[\begin{array}{ll}0 & I\end{array}\right]$} & 1.35 & 1.02 & $\mathrm{p}$ & 0.15 & 0.14 & blend & $0.21:$ & nd \\
\hline 5876 & He I & $\mathrm{p}$ & $\mathrm{p}$ & $\mathrm{p}$ & $\mathrm{p}$ & 0.16 & 0.11 & 0.24 & nd \\
\hline 4959,5007 & {$\left[\begin{array}{ll}0 & \text { III }\end{array}\right]$} & 12.17 & 12.82 & 12.94 & 1.65 & 0.88 & 0.84 & 0.86 & 0.89 \\
\hline 4861 & $\mathrm{HB}$ & 1.00 & 1.00 & 1.00 & 1.00 & 1.00 & 1.00 & 1.00 & 1.00 \\
\hline 4711,40 & {$\left[\begin{array}{ll}A & \text { IV }\end{array}\right]$} & $\mathrm{p}$ & $\mathrm{p}$ & $\mathrm{p}$ & nd & nd & blend & nd & nd \\
\hline 4686 & He II & 0.28 & 0.17 & 0.39 & $\mathrm{p}$ & $\mathrm{p}$ (blend) & $\mathrm{p}$ & nd & nd \\
\hline 4363 & {$\left[\begin{array}{ll}0 & \text { III }\end{array}\right]$} & 0.18 & 0.12 & $0.15:$ & 0.09 & \multirow{2}{*}{\} 0.39} & 0.13 & \multirow[t]{2}{*}{0.39} & \multirow[t]{2}{*}{0.46} \\
\hline 4340 & $\mathrm{H} \gamma$ & 0.32 & 0.39 & 0.35 & 0.21 & & 0.53 & & \\
\hline 4101 & H8 & 0.17 & 0.26 & 0.16 & \multirow{2}{*}{0.17} & \multirow{2}{*}{\} 0.10} & \multirow{2}{*}{0.22} & \multirow{2}{*}{\} 0.14} & nd \\
\hline 4068,76 & {$\left[\begin{array}{ll}S & I I\end{array}\right]$} & 0.41 & 0.26 & $\mathrm{p}$ & & & & & nd \\
\hline 3967,70 & {$[$ NeIII], He } & $\mathrm{p}$ & $0.37:$ & nd & nd & nd & nd & nd & nd \\
\hline 3889 & HeI, н8 & $\mathrm{p}$ & $\mathrm{p}$ & p & 0.09 & 0.11 & $0.11:$ & 0.05 & $0.10=$ \\
\hline 3869 & [NeIII] & 1.02 & 0.77 & 0.72 & & & & & \\
\hline 3726,29 & {$\left[\begin{array}{ll}0 & I I\end{array}\right]$} & 1.18 & 1.98 & 1.75 & 0.17 & 0.22 & $0.21:$ & 0.10 & nd \\
\hline 3425 & {$[\mathrm{Ne}$ V] } & 0.38 & 0.24 & 0.32 & $\mathrm{p}$ & 0.39 & 0.20 & nd & nd \\
\hline 3345 & {$[\mathrm{Ne} \mathrm{V}]$} & $0.18:$ & 0.13 & $\mathrm{p}$ & $\mathrm{p}$ & $\mathrm{p}$ & 0.07 & nd & nd \\
\hline Breadths: & & & & & & & & & \\
\hline Permitted & lines & broad & broad & $\begin{array}{r}\text { somewhat } \\
\text { broad }\end{array}$ & $\begin{array}{l}\text { very } \\
\text { broad }\end{array}$ & $\begin{array}{l}\text { very } \\
\text { broad }\end{array}$ & $\begin{array}{l}\text { very } \\
\text { broad }\end{array}$ & broad & broad \\
\hline Forbidden & lines & broad & broad & $\begin{array}{r}\text { somewhat } \\
\text { broad }\end{array}$ & broad & sharp & sharp & sharp & sharp \\
\hline
\end{tabular}


TABLE 3

ObSERVEd Line INTENSITIES Reduced to $H \beta \equiv 1$

\begin{tabular}{|c|c|c|c|c|c|c|c|c|c|c|c|}
\hline$\lambda$ & Ion & & & II & $R$ e $g$ & $g i \circ n$ & $\mathrm{TY}$ & $y p e$ & & & \\
\hline Markarian & Number & 13 & 19 & 33 & 35 & 36 & 49 & 52 & 54 & 59 & 67 \\
\hline 9545 & $\mathrm{P} 8[\mathrm{SIII}]$ & $\mathrm{p}$ & $\mathrm{p}$ & 0.97 & 1.26 & $p$ & $\mathrm{p}$ & 1.92 & nd & 0.37 & - \\
\hline 9069 & [S III] & $\mathrm{p}$ & $\mathrm{p}$ & 0.35 & 0.37 & $\mathrm{p}$ & $\mathrm{p}$ & 0.55 & nd & 0.18 & - \\
\hline 7319,30 & {$\left[\begin{array}{ll}0 & \mathrm{II}\end{array}\right]$} & nd & nd & - & 0.10 & 0.09 & nd & $\mathrm{p}$ & nd & 0.05 & nd \\
\hline 7136 & {$\left[\begin{array}{ll}A & I I I\end{array}\right]$} & $0.49:$ & 0.67 & 0.13 & 0.20 & 0.08 & nd & nd & nd & 0.08 & nd \\
\hline 6717,31 & {$\left[\begin{array}{ll}S & I I\end{array}\right]$} & 0.86 & 1.06 & 0.75 & 0.55 & 0.17 & blend & 0.62 & 1.97: & 0.22 & \\
\hline 6548,83 & [N II] & 1.51 & $0.40:\}$ & 4.79 & 0.26 & $<0.15\}$ & 3.55 & 2.06 & 6.07 & 0.15 & 4.87 \\
\hline 6562 & $\mathrm{H} \alpha$ & 4.70 & $3.90\}$ & & 3.67 & 2.96 & & 3.30 & & 2.62 & \\
\hline 6300,63 & {$\left[\begin{array}{ll}0 & \mathrm{I}\end{array}\right]$} & 0.41 & $\mathrm{p}$ & 0.17 & 0.10 & nd & 0.16 & $p$ & nd & 0.07 & \\
\hline 5876 & He I & nd & $\mathrm{p}$ & - & $0.17=$ & 0.12 & 0.10 & 0.16 & - & 0.08 & $\mathrm{p}$ \\
\hline 4959,5007 & {$\left[\begin{array}{ll}0 & \text { III }\end{array}\right]$} & 1.83 & 4.97 & 2.21 & 5.10 & 5.81 & 4.06 & 0.76 & 4.89 & 7.15 & 7.74 \\
\hline 4861 & $\mathrm{HB}$ & 1.00 & 1.00 & 1.00 & 1.00 & 1.00 & 1.00 & 1.00 & 1.00 & 1.00 & 1.00 \\
\hline 4711,40 & [A IV] & nd & nd & nd & nd & nd & nd & blend & nd & nd & nd \\
\hline 4686 & He II & $\mathrm{p}$ & $p$ & nd & nd & $0.13:$ & 0.12 & 0.21 & nd & 0.03 & nd \\
\hline 4363 & {$[0 \mathrm{III}]$} & 0.30 & 0.02 & 0.32 & 0.03 & $0.09\}$ & $0.27\}$ & 0.29 & nd & $0.06\}$ & 0.36 \\
\hline 4340 & $\mathrm{H} \gamma$ & 0.00 & 0.24 & & 0.30 & $0.39\}$ & & & nd & $0.37\}$ & \\
\hline 4101 & H8 & $0.31:$ & nd & $\mathrm{p}$ & 0.19 & 0.18 & 0.28 & $\mathrm{p}$ & nd & 0.19 & 0.33 \\
\hline 4068,76 & {$\left[\begin{array}{ll}S & I I\end{array}\right]$} & nd & nd & nd & blend & nd & nd & blend & nd & nd & \\
\hline 3967,70 & [NeIII], & $\mathrm{H} \varepsilon \quad \mathrm{nd}$ & nd & nd & $p$ & 0.26 & nd & nd & nd & 0.12 & nd \\
\hline 3889 & He I, н8 & 0.45 & $\mathrm{p}$ & 0.28 & 0.07 & $0.11\}$ & 0.17 & $0.12\}$ & nd & 0.40 & 0.74 \\
\hline 3869 & [Ne III] & & $\mathrm{p}$ & & 0.12 & $0.27\}$ & & $-\int$ & & & \\
\hline 3726,29 & {$\left[\begin{array}{ll}0 & \mathrm{II}\end{array}\right]$} & 1.47 & 2.58 & 1.92 & 1.72 & 1.24 & nd & 1.18 & 2.79 & 1.01 & 1.69 \\
\hline 3425 & {$[\mathrm{Ne} \mathrm{V}]$} & - & nd & nd & nd & nd & nd & nd & nd & nd & nd \\
\hline 3345 & [Ne V] & - & nd & nd & nd & nd & nd & nd & nd & nd & nd \\
\hline \multicolumn{12}{|c|}{ Breadths: } \\
\hline Permitted 1 & lines & sharp & sharp & sharp & sharp & sharp & sharp & sharp & sharp & sharp & sharp \\
\hline Forbidden 1 & lines & sharp & sharp & sharp & sharp & sharp & sharp & sharp & sharp & sharp & sharp \\
\hline
\end{tabular}

and Becklin 1970) and is slightly less steep than the mean slope of $3 \mathrm{C} 120$ and NGC 4151 in the 2 to $10 \mu$ region (Rieke and Low 1972). The blackbody radiation is representative of that expected from thermal reradiation from dust; the 20 to $10 \mu$ color temperature of $\mathrm{MKN} 3$ is $\sim 200 \mathrm{~K}$.

It is seen that qualitatively the observed continua do in fact show the behavior expected from the composite sources. In MKN 6, 9, 79, and 124 the powerlaw spectrum dominates in the near-infrared and contributes strongly in the visible; in all cases the $10 \mu$ fluxes are below the extrapolation of the power law by a factor between 2 and 5 . All four continua are approximately consistent with the upper curves of Figure 9; MKN 10 and 34, on the other hand, are well fitted by the bottom curve of Figure 9 in which the visible and near-infrared radiation come from stellar radiation. The ultraviolet turnup observed in MKN 9,10 , and 79 is not accounted for by this model and indicates that still another radiation component is present (see below). In MKN 1 and 3 the visible and near-infrared $(\log \nu>14)$ comes predominantly from stellar radiation; the data at $\lambda>3 \mu(\log \nu<14)$ can be represented by cool blackbody radiation.
It is significant that the two groupings in continua noted above agree with the Khachikian/Weedman classes. This general correlation is strengthened by the fact that the continua of NGC 4151 and $3 \mathrm{C} 120$, both classified as type 1 , resemble those of MKN 9 and MKN 79 while NGC 1068, when observed with an aperture larger than $2^{\prime}$, does show a composite spectrum which is similar to that of MKN 3 (Becklin and Neugebauer, private communication).

Although the correlation between the continua and the Weedman classes is strong, it should be noted that there is a range of continua present in each class. In particular, MKN 10, although it has the strong ultraviolet component characteristic of the continua of other class 1 objects, may not be dominated by the power-law spectrum while in MKN 34 there is evidence at $3 \mu$ and in the ultraviolet of excesses which can be attributed as due to the contribution by a power-law spectrum.

\section{b) Discussion}

Continua.-As described above, there is evidence that the continuum radiation observed in the program 


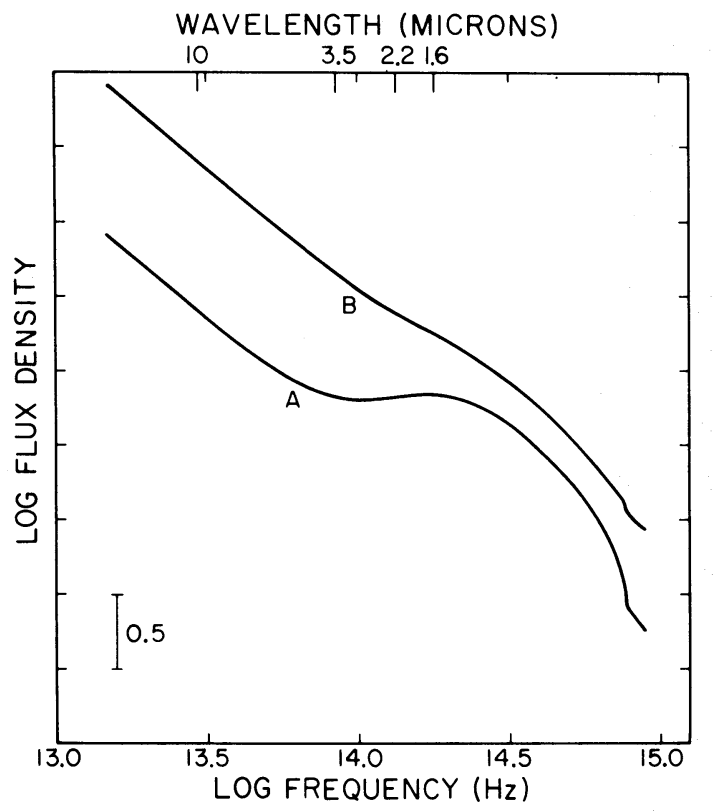

Fig. 9.-Synthetic energy distributions. The curves represent the sum of the stellar energy distribution of M31 (Sandage, Becklin, and Neugebauer 1969) plus a power-law spectrum with $f_{v} \propto v^{-1.7}$. In curve $\mathrm{A}$ the power-law flux at $\nu=10^{14} \mathrm{~Hz}$ is equal to that of M31; in curve B the powerlaw flux at $\nu=10^{14} \mathrm{~Hz}$ is 10 times that of M31.

Seyfert galaxies can have at least three distinct radiation components. The abrupt drop in flux below the $\mathrm{H}$ and $\mathrm{K}$ lines shows that the visual radiation of MKN 1, 3, and 34 contains a substantial fraction of stellar radiation. In these objects, the association of the $10 \mu$ radiation with thermal radiation from dust is made natural by the known presence of such radiation in the nearby galaxies NGC 253 and M82 (Becklin, Fomalont, and Neugebauer 1973; Rieke and Low 1975; Gillett et al. 1975) and by the evidence that the $10 \mu$ radiation in NGC 1068 is of thermal origin; Jones and Stein (1975) have reviewed the data leading to this conclusion. In addition, the slope of the continuum between $10 \mu$ and $3 \mu$ in MKN 3 is steeper than that shown by any known nonthermal source (see below). The models presented via Figures 9 and 10 show that a component with a power-law spectrum with slope -1.7 , when added to a stellar component, yields too high a $3 \mu$ flux relative to that at $10 \mu$. In contrast, the models with thermal emission from dust added to the stellar component can represent the observed continua adequately. If the dust is at a temperature of $200 \mathrm{~K}$, the linear dimensions of the dust cloud in MKN 3, for example, must exceed 10 pc. Rees et al. (1969) and Burbidge and Stein (1970) have discussed the general ramifications of thermal emission from dust in galactic nuclei.

The evidence that the smooth continua shown in Figure 6 are due to nonthermal radiation is equally indirect. The absence of a break at the $\mathrm{H}$ and $\mathrm{K}$ lines in MKN 9, 10, 79, and 124 shows that a substantial fraction of the radiation is not produced by cool stars.

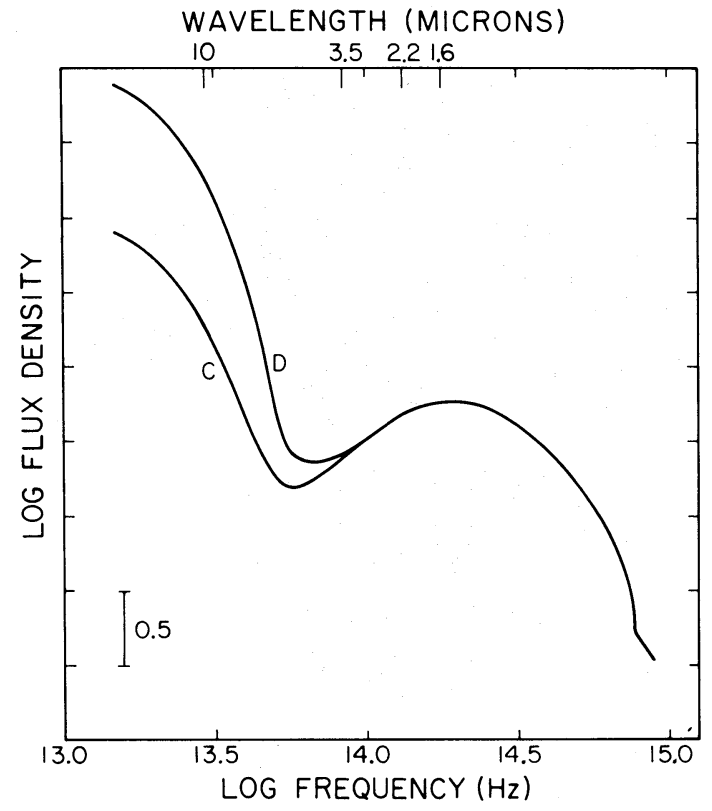

FIg. 10.- Synthetic energy distributions. The curves represent the sum of the stellar energy distribution of M31 (Sandage, Becklin, and Neugebauer 1969). plus the spectrum of a $200 \mathrm{~K}$ blackbody. In curve $\mathrm{D}$ the blackbody flux at $\nu=$ $10^{14} \mathrm{~Hz}$ is $10^{-4}$ that of $\mathrm{M} 31$; in curve $\mathrm{C}$ the blackbody flux at $\nu=10^{14} \mathrm{~Hz}$ is $10^{-5}$ that of M31.

This conclusion is substantiated by the variability discussed above in MKN 6 and by the observation of Netzer (1974) that the visible flux of MKN 79 has changed by $0.2 \mathrm{mag}$ on a time scale of 1 month. In this connection it is perhaps noteworthy that changes in the line profiles of MKN 6 have been reported by Khachikian and Weedman (1971b), Adams (1972), and Ulrich (1972). The nonzero slope eliminates the possibility of the radiation being purely from hot gas. An extension of the calculations displayed in Figures 9 and 10 shows that it is difficult to form the smooth continuum in the infrared with thermal sources without invoking dust temperatures in the range of $1000 \mathrm{~K}$. Furthermore, the slope of -1.7 is typical of the steepest of the quasi-stellar sources at wavelengths around $2.2 \mu$ (Oke, Neugebauer, and Becklin 1970) and agrees with the infrared slope of the nonstellar component of NGC 4151 which Penston et al. (1974) argue must be nonthermal on the basis of its variability. Stein and Weedman (1975) have recently argued on the basis of independent $U, V$, and $3.5 \mu$ photometry that the nonstellar component in MKN 9, 10,79 , and 124 is nonthermal.

Van der Kruit (1971) and Rieke and Low (1972) have plotted the $21 \mathrm{~cm}$ versus $10 \mu$ flux of several extragalactic objects and find a linear dependence between the two which suggests a common relationship between the infrared and radio emissions. The objects plotted include NGC 1068 which is archetypical of Seyferts with thermal $10 \mu$ emission and NGC 4151 which is archetypical of Seyfert galaxies with nonthermal $10 \mu$ continua. The significance of this relationship remains unclear. 
There are, in fact, several pieces of evidence that a fourth radiation component is present in the continua of these galaxies. If the $10 \mu$ radiation present, for example, in MKN 1, 3, and 34 is thermal radiation from dust, the infrared luminosity is substantially larger than that expected from the visual luminosity. In view of the lack of extreme reddening as measured by the $\mathrm{H} \alpha / \mathrm{H} \beta$ ratio it is therefore necessary to postulate that there is an additional ultraviolet source of radiation. The lack of reddening is also suggested by the related facts that $(a)$ there are breaks in the continua at the $\mathbf{H}$ and $\mathrm{K}$ lines and $(b)$ the observed continua are similar to those of normal unreddened galaxies. The emission lines also, if they are produced by radiation, require such a source. In the case of MKN 1, 3, and 34 there does not appear to be enough reddening present to bring the visual continuum up to a level where an extrapolation into the far-ultraviolet can account for the required ionizing flux.

For the remaining objects, the $\mathrm{H} \alpha / \mathrm{H} \beta$ ratio again suggests very little reddening. In most of these cases, however, there is a turnup or flattening in the violet which is probably caused by the postulated ultraviolet source. In summary, the arguments for a distinct ultraviolet source appear secure. This source could be hot stars or it could be a source such as bremsstrahlung or synchrotron radiation. The presence of the $[\mathrm{Ne} \mathrm{V}]$ line in Seyferts argues against the source being hot stars since [ $\mathrm{Ne} \mathrm{v}]$ is not seen in $\mathrm{H}$ II regions or in galaxies whose visual light is dominated by $\mathrm{H}$ II regions (see below).

Emission-line region.-Models of emission-line regions in Seyfert galaxies have been discussed by, inter alia, Anderson (1970); Weedman (1970); and Adams and Weedman (1975); Shields, Oke, and Sargent (1972); and Shields (1974) (3C120); Boksenberg et al. (1975) (NGC 4151); and Shields and Oke (1975) (NGC 1068).

As an idealization of a complex situation, it is generally conjectured that Seyfert galaxies contain several line-emitting regions characterized by quite different electron densities. One region, with low electron densities on the order of or less than $10^{3}$ $\mathrm{cm}^{-3}$, produces a line spectrum like that of $\mathrm{MKN}$ 1,3 , and 34 in which permitted and forbidden lines have the same profiles. Another region, which is found only in class 1 objects, has high electron densities- $N_{e} \geq 10^{8} \mathrm{~cm}^{-3}$ - and produces strong permitted lines but relatively weak forbidden lines because of the high density. The high-density clouds normally have high velocities and therefore produce very broad permitted lines while the lower density clouds tend to have smaller velocities.

An indication of the sizes of the low-density emission regions is given by the extent of the forbidden lines beyond the nuclei of MKN 1 and 3. At least in these galaxies the $[\mathrm{O}$ III] line ratios indicate an electron temperature $T_{e}=14,000 \mathrm{~K}$ and an electron density $N_{e}=10^{5} \mathrm{~cm}^{-3}$. The emissivity of the $\lambda 4959+\lambda 5007$ [O III] lines under these conditions is $10^{-15} N\left(\mathrm{O}^{++}\right)$ ergs $\mathrm{cm}^{-3} \mathrm{~s}^{-1}$. Since these galaxies emit $5 \times 10^{41}$ ergs $s^{-1}$ in these lines, the emitting volume must be $\sim 10^{55} \mathrm{~cm}^{3}$ if $N\left(\mathrm{O}^{++}\right)=10^{-3} N_{e}$; the total mass involved is $\sim 10^{60} M_{\mathrm{H}}$ or $10^{3} M_{\odot}$. If the volume filled with emitting gas were spherical, its radius would be less than 1 pc. Emission has, however, been observed at radial distances larger than $1 \mathrm{kpc}$, implying a filling factor for any plausible geometry of less than $10^{-6}$. Thus the forbidden lines apparently come from a region comprising a large fraction of the galaxy's volume sparsely filled by relatively dense filaments and wisps. Perhaps this region somewhat resembles the emitting gas region in NGC 1275 which is revealed in Lynds's (1970) monochromatic photographs.

Correlation between emission lines and continua.It was seen above that a fairly strong correlation exists between the shapes of the continua and the Khachikian/Weedman classes. Physically, we interpret the dominant distinction between class 1 and class 2 objects to be the presence in the former of a visible region consisting of clouds with electron densities $\geqslant 10^{8} \mathrm{~cm}^{-3}$ moving with very high random velocities. We also assume that the radiation components are thermal and nonthermal as discussed above. The correlation as seen would therefore imply a correlation between the strengths of the component sources of continuum radiation and the presence of observable regions of high gas density. Two possible models which can account for the correlation have been considered. In one, the explanation of class 2 objects is that a dust cloud obscures the regions of high gas density and with it the nonthermal source. This picture is unlikely, however, because of the large amount of dust needed to obscure the nonthermal sources if they are like those observed in the class 1 objects. Specifically, an examination of Table 1 shows that it would require $\sim 2 \mathrm{mag}$ at $3 \mu$ or $40 \mathrm{mag}$ of visual extinction to hide the least luminous clearly delineated nonthermal sources (MKN 6 and 79) and produce the continua of MKN 1 or 3 .

A much more likely picture is that the classes are physically controlled by the strength of the nonthermal source. In this view the nonthermal sources in class 1 objects are so bright as to hide the stellar and thermal contributions while in class 2 objects they are relatively faint. The fact that the $3 \mu$ luminosities of class 1 objects are on the average higher than those of class 2 objects supports this idea. Furthermore, in class 1 objects, the radiation from the strong nonthermal source may evaporate the dust, thus accounting for the lower ratio of $10 \mu$ to ultraviolet fluxes in class 1 relative to class 2 objects. Most importantly, this picture implies that the existence of the nonthermal source is associated with the presence of the very dense high-velocity clouds. We feel that this association is the most important conclusion of this work. This association is implicit in the discussion of Stein and Weedman (1975). An advantage of this picture is that intermediate cases with moderate-strength nonthermal sources can be easily accounted for; MKN 10 and 34 are examples where the nonthermal source is probably seen in the infrared but does not dominate the visible continuum.

It is also observed that the equivalent widths of the 
permitted lines are roughly the same in both classes (Table 2) whereas the forbidden-line equivalent widths of class 1 objects are 10 times weaker than those in class 2 objects; this thus implies that in this picture the intensities of the recombination lines emitted from the high-density region are proportional to the nonthermal flux. The intensities of the forbidden lines which are produced in the low-density regions do not change as the strength of the nonthermal flux changes. If the forbidden lines are produced in regions ionized by the nonthermal flux, this implies that the ionized region in which they are formed has a fixed volume which must be on the order of size of the galaxy. Alternatively, the forbidden lines could be produced by an ionization mechanism unrelated to the nonthermal flux. It should be recalled that the ratio of the [Ne v] to [O III] line strengths is much higher in class 1 than in class 2 objects. This is most easily explained if the forbidden-line region is in fact ionized by nonthermal radiation and implies a large increase in the far-ultraviolet ionizing flux in class 1 objects relative to class 2 sources.

\section{BLUE GALAXIES AND GALAXIES WITH NUCLEAR H II REGIONS}

a) Phenomenology $(M K N$ 13, 19, 33, 35, $36,49,52,54,59,67)$

The remainder of the program galaxies have narrow emission lines; and the emission, as will be discussed below, appears to come from $\mathrm{H}$ II regions either in the nucleus or extended over a significant part of the galaxy. For these objects, there is no reason to suppose that the Balmer lines are formed by any process other than recombination. In the cases where the $\mathrm{H} \alpha / \mathrm{H} \beta$ ratio is observed, the reddening is assumed to be that required to make the ratio 2.95 . The reddeningcorrected continua, using Whitford's (1948) reddening curve, are included in Figures 7 and 8. In those cases where the ratio of $\mathrm{H} \alpha$ to $\mathrm{N}$ II was not observed, it was assumed, rather arbitrarily, that the $\mathrm{N}$ II line strength before reddening correction was 0.40 relative to $\mathrm{H} \beta$. In the case of MKN 59, where the observed $\mathrm{H} \alpha / \mathbf{H} \beta$ ratio is only 2.63 , no corrections were made.

The light from these galaxies, in contrast to that of those classified as Seyfert galaxies, comes from diffuse irregular patches with high surface brightness. Generally there is no pronounced central condensations. Some of the objects (e.g., MKN 59) appear on direct plates to be very extended individual $\mathrm{H}$ II regions within an irregular galaxy. Others (e.g., MKN 36) appear to be "isolated extragalactic regions" like I Zw $18=$ MKN 116 discussed by Sargent and Searle (1970). MKN 52 is an isolated example of a galaxy with narrow emission lines confined to a bright nucleus. MKN $54=\mathrm{HZ} 46$ is a very much elongated galaxy of high surface brightness.

The extent of the region over which radiation is emitted is often sufficient to result in a significant aperture dependence of the photometry over the apertures used. As a result of this plus the lack of central condensations, intercomparisons between data obtained with different instruments and at different times is often difficult, if not meaningless, since positioning is not easily reproducible. MKN 33 and 35 are probably the most extreme cases giving measurements which depend on aperture size and position; but all the galaxies of this group suffer this problem, and any intercomparison of results obtained with different techniques must be viewed with extreme care.

Despite the ambiguities caused by the extended nature of these galaxies, the continua shown in Figures 7 and 8 are found, with the exception of MKN 52 and 54 , to be essentially constant in flux density per unit frequency interval from 0.3 to $2.2 \mu$ after corrections for reddening have been applied. In the case of MKN 52 the different nature of the continuum is real, but in the case of MKN 54 the reddening correction is very uncertain because the emission lines are weak and only one slit spectrum is available. In cases like MKN $13,19,33$, and 67 the Balmer jump in absorption is clearly present while in MKN 52 the absorption break near the $\mathrm{H}$ and $\mathrm{K}$ lines dominates the continuum.

The $10 \mu$ fluxes have been measured definitely only for MKN 33 and 52, although a marginal detection was obtained in MKN 67. For MKN 59, a limit (3 standard deviations) of $\log f_{v}\left(\right.$ ergs s $\mathrm{s}^{-1} \mathrm{~cm}^{-1}$ $\left.\mathrm{Hz}^{-1}\right)=-24.6$ at $10 \mu$ was obtained. Thus all measurements are consistent with there being an excess flux at $10 \mu$ a factor of 4-10 above the extrapolated flat continuum.

The spectra of the galaxies in this group all have narrow emission lines. In contrast to the Seyfert galaxies which generally show lines of [Ne v] and $\left[\mathrm{O}_{\mathrm{I}}\right],[\mathrm{Ne} \mathrm{v}]$ is absent in all objects and [O $\left.\mathrm{I}\right]$ is weak or absent. For all but MKN 52, the emission-line ratios and correlations among them follow the relations which Searle (1971) found to obtain for H II regions in the outer spiral arms of Sc galaxies. MKN 52 is apparently an example of a galaxy containing nuclear $\mathrm{H}$ il regions. The object has a bright nucleus whose spectrum shows slightly widened lines (full width at half-maximum $=60 \mathrm{~km} \mathrm{~s}^{-1}$ ). The spectrum is of low excitation and is unique among the program objects in having $\lambda 5007$ of [O III] weaker than $\lambda 3727$ of [O II]. Its spectrum is, however, like those of $\mathrm{H}$ II regions in the inner arms of spiral galaxies (Searle 1971). Another galaxy with bright nuclear regions showing similar spectra is NGC 1808 (Arp and Bertola 1970). An abundance analysis of MKN 35, 36, and 59 , which are the three blue galaxies for which the necessary information is available, shows all three to be oxygen- and neon-poor. Oxygen and neon are the only elements that can be studied other than $\mathrm{H}$ and He. MKN 35 and 59 are oxygen-poor by a factor of 2 ; MKN $36=$ Haro 4 is oxygen-poor by a factor of 4 with respect to $\mathrm{H}$ iI regions in the solar neighborhood. In this respect too the blue galaxies share the characteristics of I Zw 18 and II Zw 40 (Searle and Sargent 1972).

\section{b) Discussion}

The fact that an appreciable fraction of the Markarian galaxies can be classified as "blue galaxies" 
has been recognized by several authors (see, e.g., Weedman and Khachikian 1968, 1969; Sargent 1972; Weedman 1973); in fact, several of the program galaxies were included in the pioneering list of blue galaxies by Haro (1956).

The spectroscopic data presented here do not add significantly to the understanding of these blue galaxies beyond the detailed description of I $\mathrm{Zw}$ 18 and II Zw 40 by Searle and Sargent (1972). The energy distributions do show, however, that the constancy of the spectrum out to $2.2 \mu$ is a common property of these regions, although the origin of the $2.2 \mu$ flux is in doubt. On the basis of the observed $\mathrm{H} \beta$ line intensities the observed $2.2 \mu$ flux density exceeds, for reasonable temperatures, that expected if the radiation were produced by thermal bremsstrahlung by a factor of at least 10. The $2.2 \mu$ flux could arise from $M$ and $K$ supergiant stars and dust which are present along with the young ionizing stars, or it could arise from a population of old $M$ and $K$ giant stars. The relative uniformity of the energy distributions is consistent with the first hypothesis. For it to be consistent with the second hypothesis it is necessary for the present rate of star formation to be the same fraction of past star formation for all blue galaxies. This could come about, for example, if these blue galaxies experience a uniform rate of star formation as suggested by Searle, Sargent, and Bagnuolo (1973). For MKN 52 the star formation at the present time is probably less important; from the slope of the spectrum and the break near the $H$ and $K$ lines most of the radiation contributing to the continuum is probably coming from an old population in the nucleus.

The $10 \mu$ fluxes present in MKN 33, 34, 52, and 67 can be most easily interpreted as arising from thermal emission from dust at a temperature around $200 \mathrm{~K}$. It should be emphasized that the $10 \mu$ measurements of this class of Markarians are preliminary and do not form a complete survey but that $10 \mu$ flux has been detected in the four galaxies that have been observed. The emission from these galaxies shows a strong similarity to galactic $H$ II regions. In particular, the $10 \mu$ fluxes of the four Markarian galaxies studied are apparently greater than or equal to those predicted from the available ionizing flux on the basis of the generally applicable relationship between the infrared and the ionizing fluxes described by Wynn-Williams and Becklin (1974) for galactic $\mathrm{H}$ II regions; the number of available ionizing photons has been estimated from the $\mathrm{H} \beta$ flux. In contrast, of $14 \mathrm{H}$ II regions associated with nearby galaxies and studied by Strom et al. (1974) only in one case, at most, does the infrared flux agree with the relationship; the rest are apparently deficient in $10 \mu$ flux. The $\mathrm{H}$ II regions of the present program are thus apparently differentiated from those studied by Strom et al. (1974) by their large $10 \mu$ flux. The Markarian galaxies are farther away than the $\mathrm{H}$ II regions observed by Strom et al., and spatial resolution effects might be important.

\section{SUMMARY}

Markarian galaxies which show emission lines can be divided into those which can be considered Seyfert galaxies and those which are extragalactic $\mathrm{H}$ in regions.

The photometry, spectra, and broad-based spectrophotometry of the Seyfert galaxies from the ultraviolet through the infrared broadly confirm the ideas that there are physically two types of Seyferts which generally agree with the classes defined by Khachikian and Weedman (1971a). Class 1 Seyferts are characterized by having a high-velocity region of gas with density greater than $10^{8} \mathrm{~cm}^{-3}$ which is missing or possibly obscured in class 2 objects. Additionally, in class 1 objects the continuum is usually dominated by nonthermal radiation while in class 2 objects the continuum is dominated by stellar radiation plus an infrared component which is presumably thermal radiation from dust. Finally, an important conclusion of this work is that the presence of broad emission lines and the presence of dominating nonthermal continuum are closely associated.

The remaining Markarian galaxies of those studied here are examples of extragalactic $H$ II regions as described by Sargent and Searle (1970). The $10 \mu$ observations of these galaxies are consistent with the presence of thermal emission from dust.

We want to thank the night assistants of the Hale telescope, Gary Tuton and Juan Carrasco, for their help in obtaining these data as well as G. Garmire who collaborated in making the early infrared measurements and B. Turnrose and K. Matthews for their assistance. G. N. thanks the Guggenheim Foundation for a sabbatical fellowship and the Institute of Astronomy, Cambridge, for their hospitality; discussions with M. J. Rees were most helpful. We also thank J. Bennett and S. Hage for their help in preparing the data and this manuscript. Funds were provided by National Science Foundation grant MPS 74-18555 and by National Aeronautics and Space Administration grants NGL 05-002-207 and NGL 05-002-134.

\section{REFERENCES}

Adams, T. F. 1972, Ap. J. (Letters), 172, L101.

Adams, T. F., and Weedman, D. W. 1975, Ap. J., 199, 19. Anderson, K. S. 1970, Ap.J., 162, 743.

Arakelian, M. A., Dibay, E. A., and Yesipov, V. F. 1970, Astrofizika, 6, 39.

Arp, H., and Bertola, F. 1970, Ap. Letters, 6, 65.

Arp, H. C., Khachikian, E. Y., Lynds, C. R., and Weedman, D. W. 1968, Ap. J. (Letters), 152, L103.

Bahcall, J. N., and Kozlovsky, B. 1969, Ap. J., 155, 1077.
Becklin, E. E., Fomalont, E. B., and Neugebauer, G. 1973, Ap. J. (Letters), 181, L27.

Boksenberg, A., Shortridge, K., Allen, D. A., Fasbury, R. A. E., Penston, M. V., and Savage, A. 1975, preprint.

Burbidge, G. R., and Stein, W. A. 1970, Ap. J., 160, 573.

Gillett, F. C., Kleinmann, D. E., Wright, E. L., and Capps, R. W. 1975, Ap. J. (Letters), 198, L65.

Haro, G. 1956, Bol. Obs. Tonantzintla y Tacubaya, No. 14, p. 8. 
Jones, T. W., and Stein, W. A. 1975, Ap. J., 197, 297.

Khachikian, E. Ye., and Weedman, D. W. 1971a, Astrofizika, 7, 389. 1971b, Ap. J. (Letters), 164, L109.
1974, Ap. J., 192, 581.

Lynds, R. 1970, Ap. J. (Letters), 159, L151.

Markarian, B. E. 1967, Astrofizika, 3, 55. 1969 a , ibid., 5, 443.

$1969 b$, ibid., p. 581

1972, ibid., 8, 165.

Markarian, B. E., and Lipovetsky, V. A. 1971, Astrofizika, 7,511 .

. 1972 , ibid, 8,155 .

Netzer, H. 1974, M.N.R.A.S., 167, 1P

Oke, J. B., Neugebauer, G., and Becklin, E. E. 1970, Ap. J., 159,341

Oke, J. B., and Schild, R. E. 1970, Ap. J., 161, 1015.

Osterbrock, D. E., and Parker, R. A. R. 1965, Ap.J., 141, 892.

Penston, M. V., Penston, M. J., Selmes, R. A., Becklin, E. E., and Neugebauer, G. 1974, M.N.R.A.S., 169, 357

Rees, M. J., Silk, J. I., Werner, M. W., and Wickramasinghe, N. C. 1969 , Nature, 223, 788 .

Rieke, G. H., and Low, F. J. 1972, Ap. J. (Letters), 176, L95.

. 1975, Ap. J., 197, 17.

Sandage, A. R., Becklin, E. E., and Neugebauer, G. 1969, Ap. J., 157, 55

Sargent, W. L. W. 1972, Ap. J., 173, 7.
Sargent, W. L. W., and Searle, L. 1970, Ap. J. (Letters), 162, L155.

Searle, L. 1971, Ap. J., 168, 327.

Searle, L., and Sargent, W. L. W. 1972, Ap. J., 173, 25.

Searle, L., Sargent, W. L. W., and Bagnuolo, W. G. 1973, Ap. J., 179, 427.

Shields, G. A. 1974, Ap. J., 191, 309.

Shields, G. A., and Oke, J. B. 1975, Ap. J., 197, 5.

Shields, G. A., Oke, J. B., and Sargent, W. L. W. 1972 , Ap. J., 176, 75.

Stein, W. A., and Weedman, D. W. 1975, preprint.

Strom, S. E., Strom, K. M., Grasdalen, G. L., and Capps, R. W. 1974, Ap. J. (Letters), 193, L7.

Ulrich, M.-H. 1972, Ap. J. (Letters), 171, L35.

van der Kruit, P. C. 1971, Astr. and Ap., 15, 110.

Wampler, E. J. 1971, Ap. J., 164, 1.

Wampler, E. J., and Oke, J. B. 1967, Ap. J., 148, 695.

Weedman, D. W. 1970, Ap. J., 159, 405.

. 1973, Ap. J., 183, 29.

Weedman, D. W., and Khachikian, E. Ye. 1968, Astrofizika, $4,587$.

4, 587. 1969, ibid., 5, 113.

Whitford, A. E. 1948, Ap. J., 107, 102.

Wilson, W. J., Schwartz, P. R., Neugebauer, G., Harvey, P. M., and Becklin, E. E. 1972, Ap. J., 177, 523.

Wynn-Williams, C. G., and Becklin, E. E. 1974, Pub. A.S.P., 86, 5 .

E. E. Becklin and G. Neugebauer: Downs Laboratory of Physics (320-47), California Institute of Technology, Pasadena, CA 91125

J. B. OKE: Hale Observatories, California Institute of Technology, Pasadena, CA 91125

L. SEARLE: Hale Observatories, 813 Santa Barbara Street, Pasadena, CA 91101 